\title{
Efecto de la propagación asexual y prolongación del período vegetativo de Morus alba en la producción de capullos de seda
}

\author{
Alicia Pelicanoํㅗ Marta Divo de Sesar, Norma Zamuner, José Luis Danelón \\ y Miriam Yoshida \\ Facultad de Agronomía, Universidad de Buenos Aires \\ (C1417DSE) Av. Martín 4453, Buenos Aires, Argentina
}

\begin{abstract}
A. Pelicano, M. Divo de Sesar, N. Zamuner, J. L. Danelón, and M. Yoshida. 2007. The effect of asexual propagation and persistence of the vegetative growth of Morus alba on silk cocoon production. Cien. Inv. Agr. 34 (2):81-89. The goal of this work was to establish an efficient protocol for vegetative propagation of Morus alba. At the same time this study aimed to evaluate the influence of this propagation protocol on the feeding of the larvae of Bombyx mori and crude silk production. Vegetatively propagated mulberry plants were transplanted to $4 \mathrm{~L}$ containers and treated with $15 \mathrm{~g}$ of fertilizer, N:P:K (15:15:15,), and/or 6-benzyladenine $\left(5 \mathrm{mg} \cdot \mathrm{L}^{-1}\right)(\mathrm{Ck})$. In autumn and spring, leaves were used for the feeding of one cohort of third stage larvae. It was observed that $60 \%$ of the plants treated with $\mathrm{Ck}$ sprouted before the others, in July, and the rest in August. Although the fertilized plants (TF) and those fertilized and treated with 6-benzyladenine (TFCk) sprouted shortly before the control (T), the Ck treated plants were notably uniform and had more and larger leaves. In the TFCk treatment, the percentage of fiber, insoluble in neutral detergent (FIND) free from ash, was minor $(29.73 \%)$. The quality of the food was reflected in the weight gain and in crude silk percentage. Although interaction between $\mathrm{TF}$ and $\mathrm{Ck}$ was not significant, there were significant differences between control plants $(\mathrm{T})$ and those treated with TF, TCk and TFCk. In the autumn test, the TCk treatment negatively affected the larvae of $B$. mori. These larvae twisted, assiduously inducing intestine evacuation. This caused reduced food consumption, and it was a transitory effect, but that meant a lower final weight. The results of this work are very promising and useful for massive production since a longer vegetative cycle for $M$. alba would allow a greater number of $B$. mori generations per year.
\end{abstract}

Key words: Bombyx mori, cytokinins, Morus alba, mulberry.

\section{Introducción}

La sericicultura se define como la cría y aprovechamiento del gusano de seda (Bombyx mori L., Lepidoptera: Bombicidae), criado en cautiverio para obtener seda natural con fines comerciales. Esta es una alternativa de diversificación productiva que tiene gran importancia socioeconómica, por la elevada

Recibido 27 de marzo 2006. Aceptado 14 de marzo 2007.

' Dirigir correspondencia a A. Pelicano: pelicano@agro.uba.ar utilización de mano de obra, especialmente la del grupo familiar. Se considera un agronegocio de especialidades, con alto valor agregado, que posibilita la generación de productos tanto para el mercado interno como para la exportación (Acerbi et al., 2005).

Si bien existen algunos emprendimientos de sericicultura en distintas zonas de Argentina, estos aún no generan producciones a nivel industrial. Los factores prioritarios a considerar incluyen la producción de huevos, capacitación para el buen manejo de la cría, obtención de 
productos de excelente calidad y provisión de moreras (Morus alba L.) (Acerbi et al., 2005). El productor que inicie esta actividad deberá considerar que en la producción de capullos, generalmente los costos superan a los ingresos. Sin embargo, el producto elaborado, es decir, la tela de seda natural y/o una prenda, presenta un alto precio final (Acerbi et al., 2005).

El gusano de seda es un insecto de metamorfosis completa. Habitualmente se cría bajo cubierta durante la primavera y el verano. El estado larval dura entre 20 y 25 días, posteriormente teje el capullo en el cual forma la crisálida. Finalmente emerge el adulto al término de 14 días. El capullo está formado por un único hilo de seda cruda de 800 a 1.500 m de longitud. Ello está condicionado por la raza, la alimentación y las condiciones de cría (Cifuentes y Schon, 1998).

Esta especie puede presentar uno, dos o más ciclos de vida por año. Para la actividad de cría, se suelen utilizar poblaciones que desarrollan varias generaciones anuales. Estas permiten obtener rendimientos de capullos significativamente más importantes (Cifuentes y Schon, 1998).

Se trata de un insecto monófago que se alimenta exclusivamente de hojas de morera (Morus sp). Durante su etapa juvenil, el cuerpo del gusano de seda aumenta en peso, aproximadamente 10.000 veces. Por esto come vorazmente, sobre todo en la última etapa de su desarrollo (quinto estadio larvario), en la que ingiere cerca de 500 veces más hojas que al nacimiento (Cifuentes y Schon, 1998). La calidad del alimento influye considerablemente en su desarrollo como así también afecta la producción de seda bruta (Pelicano et al., 2004).

En Argentina, la morera se desarrolla naturalmente desde los $22^{\circ}$ hasta los $38^{\circ}$ de latitud S. En una zona geográfica tan amplia, existen distintas condiciones agroecológicas que determinan períodos vegetativos de variada duración, condición que limita el número de generaciones por año. A la latitud de Buenos Aires las moreras brotan aproximadamente a mediados de septiembre completando el ciclo de crecimiento vegetativo anual a fines de marzo, tiempo que permite realizar entre dos y tres crías anuales. Es importante contar con hojas de morera de buena calidad por un período de tiempo prolongado para aumentar el número de ciclos anuales y, por lo tanto, la producción de capullos.

La propagación de morera, con fines productivos, se realiza a través de la multiplicación vegetativa. Este proceso se puede repetir desde la primavera hasta fin del verano. En ensayos con otras especies, se observó que aplicaciones de 6-bencilaminopurina (BAP), una citoquinina, durante la fase final del enraizamiento aumenta el porcentaje del mismo (en invierno tardío y primavera temprana), calidad de raíces, número y largo de las ramificaciones (Divo de Sesar et al., 2005). Este efecto se prolonga durante la etapa de vivero existiendo interacción entre auxinas, citoquininas y/o fertilizantes (Divo de Sesar et al., 2003). Si bien, este regulador de crecimiento participa en diferentes funciones dentro de las plantas, su efecto más importante es sobre el retraso de la senescencia (Thomas et al., 2003)

Los objetivos de este trabajo fueron: 1. Elaborar un protocolo de propagación asexual para M. alba; 2. Producción de plantas con mayor duración del período vegetativo; 3. Evaluar el efecto de diferentes estrategias de manejo de moreras sobre el desarrollo de las larvas de $B$. mori y sobre la producción seda cruda.

\section{Materiales y métodos}

\section{Propagación de moreras}

Con este propósito se obtuvieron estaquillas semileñosas, apicales y subapicales, de $6-8 \mathrm{~cm}$ de longitud, con al menos dos nudos, con hojas. Estas se obtuvieron de brotes del crecimiento primaveral-estival del año, de plantas madres

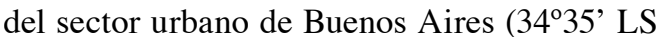
y $58^{\circ} 29^{\prime}$ LO). Todas las estacas se trataron con benomil $\left(1,5 \mathrm{~g} \cdot \mathrm{L}^{-1}\right.$, Benlate ${ }^{\circ}$, Du Pont de Nemours and Company Agricultural Products, EUA), y parte de estas estacas se trataron con ácido indolbutírico (IBA) adsorbido en talco

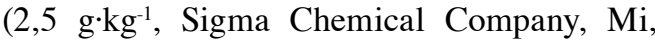
EUA). Estacas igualmente tratadas con benomil pero sin aplicación de IBA se mantuvieron 
como testigos. Las estacas ( 3 repeticiones y 100 estacas por repetición, para cada tratamiento) se plantaron en mesas de enraizamiento a temperatura ambiente, en un sustrato de perlita (grava volcánica expandida, de $8 \mathrm{~cm}$ de altura), con una densidad de 800 estacas por $\mathrm{m}^{2}$. Se mantuvieron con riego intermitente según la demanda ambiental.

A las ocho semanas finalizó el período de enraizamiento para iniciar la etapa de establecimiento y adaptación. Las estaquillas enraizadas provenientes de la concentración $2500 \mathrm{mg} \cdot \mathrm{kg}^{-1}$ de IBA se transplantaron a macetas de cloruro de polivinilo de $4 \mathrm{~L}$ con un sustrato compuesto por tierra, perlita, arena y cáscara de arroz (3:1:1:1) para su adaptación final. Cada maceta se fertilizó con $15 \mathrm{~g}$ de $\mathrm{N}$ : $\mathrm{P}: \mathrm{K}$ (15:15:15, v:v:v:) y en tres oportunidades cada cinco días se asperjaron con $5 \mathrm{mg} \cdot \mathrm{L}^{-1} \mathrm{de}$ 6-bencilaminopurina (BAP, Sigma Chemical Company, Mi, EUA), empleando $1 \mathrm{ml}$ por planta. Por lo tanto, los tratamientos fueron: 1. Testigo (T), Plantas sin tratar; 2. Plantas tratadas con BAP (TCk), 3. Plantas fertilizadas sin tratar con BAP (TF) y 4. Plantas fertilizadas y tratadas con BAP (TFCk) (55 plantas por tratamiento).

Las plantas se mantuvieron en invernadero, a temperatura ambiente, en condiciones de semi sombra, 230 a 280 watts $\cdot \mathrm{m}^{-2}$, el desmalezado se realizó a mano y se regaron cada dos días. Se efectuaron mediciones, cada dos semanas, hasta la caída de las hojas, tomando 10 plantas al azar de cada tratamiento. Durante dos ciclos de crecimiento consecutivos, cada 2 semanas, se evaluó el número de plantas brotadas.

Las muestras de hojas de morera se secaron en estufa con circulación forzada de aire $\left(\right.$ a $55^{\circ} \mathrm{C}$ hasta peso constante) se molieron y se tamizaron en malla de $1 \mathrm{~mm}$ (Wiley Mill, Arthur $\mathrm{H}$. Thomas, PA, EUA). Se determinó el contenido de materia seca (MS), proteína bruta (PB) y fibra insoluble en detergente neutro (FIDN) libre de cenizas (Van Soest et al., 1991).

\section{Bioensayos con gusanos de seda}

Se realizaron ensayos en otoño (marzo-abril) y primavera (octubre-noviembre) de 2005. Con este propósito se utilizó una cohorte de larvas de tercer estadio de B. mori, raza china, ya que es a partir de esta fase que se estabiliza la supervivencia y comienza a hacerse evidente la ganancia de peso. Las larvas fueron alimentadas 3 a 4 veces por día con hojas jóvenes de morera, provenientes de los cuatro tratamientos aplicados $(\mathrm{n}=112)$. Cada 4 días se realizaron los cambios de las cajas de cría, desinfectándolas con formaldehído adsorbido en talco (10\%, Sigma Chemical Company, Mi, EUA). Se evaluó la ganancia de peso (peso de la larva al momento de encapullado menos el peso de la larva al inicio del ensayo) y porcentaje de seda cruda (peso del capullo sin pupa $\mathrm{x}$ peso de capullo con pupa $^{-1}$ x 100).

\section{Diseño y análisis estadísticos}

Los resultados obtenidos con morera se evaluaron a través de análisis de varianza de acuerdo a un modelo completamente al azar con estructura factorial. Para porcentaje de enraizamiento los factores fueron tratamiento hormonal y momento de evaluación $(2 \times 2)$. Para la evolución del número de yemas y hojas a través del tiempo los factores fueron tratamiento a las plantas y momento de evaluación ( 4 x 3). Para número de hojas, peso fresco de hojas y peso fresco total los factores fueron tratamiento a las plantas $y$ momento del corte ( $4 \times 2$ ). Los promedios se separaron según la prueba de Tukey. Cuando se emplearon porcentajes, previamente, los valores se transformaron según el modelo de arco-seno para proporciones. Se utilizó el software NCSS (Hintze, 1991).

Las larvas se distribuyeron con un diseño totalmente aleatorizado con estructura factorial ( 2 x 4). Los factores fueron época de ensayo y tratamiento a plantas en cajas plásticas (22 x 15 x $4 \mathrm{~cm}$ ), empleando siete larvas por caja, con cuatro repeticiones por tratamiento. Con los resultados obtenidos se realizó un análisis de varianza según un modelo factorial y los promedios se separaron según la prueba de Tukey, utilizandoel software estadístico InfoStat (InfoStat 2002. Grupo InfoStat. Facultad de Ciencias Agropecuarias, Universidad Nacional de Córdoba. Córdoba, Argentina) (Di Rienzo et al., 2002). 


\section{Resultados y discusión}

\section{Propagación de morera}

Las mejores respuestas al enraizamiento se obtuvieron con brotes jóvenes tratados con IBA (Cuadro 1), con un alto porcentaje de enraizamiento en el primer mes (80\%), siendo innecesario prolongar los ensayos de enraizamiento para evitar la pérdida de calidad de las plántulas logradas (Divo de Sesar et al., 2003). La suplementación con citoquininas y/o fertilizantes mejoró la productividad (número y tamaño de hojas) (Cuadros 2 y 3) y la calidad de las plántulas obtenidas, estimada por el contenido de proteína (Cuadro 4).

Reguladores de crecimiento citoquinínicos tienen importancia en varias fases del desarrollo y crecimiento de las plantas, incluyendo la desinhibición y diferenciación de yemas, alargamiento celular, flujo de asimilados y nutrientes a través de la planta. Si bien, el efecto más extraordinario ocurre sobre la senescencia; habiéndose previamente observado interacción entre fertilizantes y citoquininas (Thomas et al., 2003; Divo de Sesar, 2005). Sin embargo, la interacción entre fertilizantes y citoquinina no fue significativa en estos ensayos.
Cuadro 1. Efecto del ácido indolbutírico (IBA) sobre la proporción de estaquillas enraizadas de Morus alba, determinado luego de 4 y 8 semanas post plantación.

Table 1. Effect of indolbutyric acid (IBA) on the proportion of rooting obtained with cuttings of Morus alba four and eight weeks post planting.

\begin{tabular}{ccc}
\hline \multirow{2}{*}{$\begin{array}{c}\text { IBA } \\
\mathrm{mg} \cdot \mathrm{kg}^{-1}\end{array}$} & \multicolumn{2}{c}{ Enraizamiento, \% } \\
\hline 0 & 4 semanas & 8 semanas \\
\hline 2500 & $43 \mathrm{~b}^{1}$ & $61 \mathrm{~b}^{1}$ \\
\hline
\end{tabular}

'Promedios de tres repeticiones, 100 estacas por repetición. Los datos fueron transformados con arco seno previo al análisis estadístico. Promedios seguidos por letras diferentes dentro de cada columna son significativamente diferentes según Tukey $(\mathrm{p} \leq 0,05)$.

${ }^{\prime}$ Means of three replicates each consisting of 100 cuttings. Data were transformed to arcsine before statistical analysis. Means followed by different letters within each column are significantly different according to Tukey $(p \leq 0.05)$.

Si bien las plántulas enraizadas se asignaron al azar, el calibre (diámetro) de las estacas fue significativamente menor en las plántulas asignadas para el tratamiento con TCk a inicio del ensayo (18 de febrero). El efecto de este tratamiento sobre la altura de la planta, número de hojas y número de yemas no fue estadísticamente significativo.

Es importante considerar que los tallos gruesos

Cuadro 2. Efecto del regulador (6-benciladenina) de crecimiento y de la fertilización (N:P:K) sobre la evaluación de las yemas y el número de hojas por planta de morera (Morus alba).

Table 2. Effect of the growth regulator (6-benzyladenine) and fertilization (N:P:K) on the evolution of buds and leaves of Morus alba plants.

\begin{tabular}{llccc}
\hline Órganos & \multicolumn{1}{c}{ Tratamientos $^{1}$} & \multicolumn{3}{c}{ Evaluaciones realizadas el: } \\
& & 18-febrero & 01-marzo & 29 -marzo \\
\hline Yemas, no. & Testigo (T) & $4,11 \mathrm{a}^{2}$ & $5,16 \mathrm{c}^{2}$ & $7,21 \mathrm{c}^{2}$ \\
& Regulador de crecimiento (TCk) & $4,61 \mathrm{a}$ & $7,59 \mathrm{~b}$ & $9,80 \mathrm{ab}$ \\
& Fertilizante (TF) & $4,88 \mathrm{a}$ & $8,95 \mathrm{a}$ & $8,87 \mathrm{~b}$ \\
TCk + TF & $3,98 \mathrm{a}$ & $8,05 \mathrm{~b}$ & $10,71 \mathrm{a}$ \\
Hojas, no. & Testigo (T) & $2,48 \mathrm{a}^{2}$ & $4,70 \mathrm{c}^{2}$ & $6,07 \mathrm{c}^{2}$ \\
& Regulador de crecimiento (TCk) & $2,59 \mathrm{a}$ & $5,61 \mathrm{~b}$ & $7,89 \mathrm{~b}$ \\
& Fertilizante (TF) & $3,14 \mathrm{a}$ & $7,21 \mathrm{a}$ & $7,23 \mathrm{bc}$ \\
& TCk + TF & $2,79 \mathrm{a}$ & $6,84 \mathrm{a}$ & $9,09 \mathrm{a}$ \\
\hline
\end{tabular}

${ }^{1}$ Regulador de crecimiento $=1 \mathrm{~mL}$ por planta de una solución de 6 -bencilaminopurina (BAP) $\left(5 \mathrm{mg} \cdot \mathrm{L}^{-1}\right)$. Fertilizante $=15 \mathrm{~g}$ por planta de $\mathrm{N}: \mathrm{P}: \mathrm{K}(15: 15: 15$, v:v:v:)

${ }^{2}$ La interacción entre fertilizante y regulador de crecimiento no fue estadísticamente significativa a p $\leq 0,05$, tanto para el número de yemas como para el número de hojas. Promedios seguidos por letras diferentes dentro de cada columna son significativamente diferentes según Tukey $(\mathrm{p} \leq 0,05)$

${ }^{I}$ Growth regulator $=1 \mathrm{~mL}$ per plant of a $5 \mathrm{mg} \cdot \mathrm{L}^{-1}$ solution of 6-benzyladenine (BA). Fertilizer $=15 \mathrm{~g}$ per plant of an $\mathrm{N}: P: \mathrm{K}$ mixture $(15: 15: 15$, v:v:v). ${ }^{2}$ The interaction between fertilizer and growth regulator was not statistically significant at $p \leq 0.05$, both for buds and leaves. Means followed by different letters within each column are significantly different according to Tukey $(p \leq 0.05)$. 
Cuadro 3. Efecto del regulador (6-benciladenina) de crecimiento y de la fertilización (N:P:K) sobre el número de hojas por planta, peso foliar fresco y peso fresco por planta de Morus alba, determinado en otoño y primavera.

Table 3. Effect of the growth regulator (6-benzyladenine) and fertilization (N:P:K) on the number of leaves per plant, leaf fresh weight and total fresh weight per white mulberry (Morus alba) plant, determined in the fall and spring.

\begin{tabular}{|c|c|c|c|}
\hline Tratamientos $^{1}$ & $\begin{array}{c}\text { Hojas por planta } \\
\text { no. }\end{array}$ & $\begin{array}{c}\text { Hoja } \\
\text { peso fresco } \\
\mathrm{g}\end{array}$ & $\begin{array}{c}\text { Planta } \\
\text { peso fresco total } \\
\mathrm{g}\end{array}$ \\
\hline \multicolumn{4}{|c|}{ Otoño } \\
\hline Testigo (T) & $5,64 \mathrm{c}^{1}$ & $0,80 \mathrm{~b}^{1}$ & $4,51 \mathrm{~b}^{1}$ \\
\hline Regulador de crecimiento (TCk) & $7,32 a b$ & $1,41 \mathrm{a}$ & 10,32 a \\
\hline Fertilizante (TF) & $6,14 \mathrm{~b}$ & $0,96 \mathrm{~b}$ & $5,89 \mathrm{~b}$ \\
\hline $\mathrm{TCk}+\mathrm{TF}$ & 7,92 a & $1,17 \mathrm{ab}$ & 9,26 a \\
\hline \multicolumn{4}{|c|}{ Primavera } \\
\hline Testigo (T) & $6,95 \mathrm{~b}$ & $0,96 \mathrm{~b}$ & $6,67 \mathrm{bc}$ \\
\hline Regulador de crecimiento (TCk) & $9,20 \mathrm{a}$ & $1,54 \mathrm{a}$ & 14,17 a \\
\hline Fertilizante (TF) & $6,55 \mathrm{~b}$ & $1,14 \mathrm{~b}$ & $7,47 \mathrm{~b}$ \\
\hline $\mathrm{TCk}+\mathrm{TF}$ & $7,12 \mathrm{~b}$ & $1,23 \mathrm{ab}$ & $8,75 \mathrm{~b}$ \\
\hline
\end{tabular}

${ }^{1}$ Regulador de crecimiento $=1 \mathrm{~mL}$ por planta de una solución de 6-bencilaminopurina $(\mathrm{BAP})\left(5 \mathrm{mg} \cdot \mathrm{L}^{-1}\right)$. Fertilizante $=15 \mathrm{~g}$ por planta de $\mathrm{N}: \mathrm{P}: \mathrm{K}(15: 15: 15, \mathrm{v}: \mathrm{v}: \mathrm{v}:)$.

${ }^{2} \mathrm{La}$ interacción entre fertilizante y regulador de crecimiento no fue estadísticamente significativa a $\mathrm{p} \leq 0,05$, tanto para el número de hojas, peso foliar fresco como para el peso fresco por planta. Promedios seguidos por letras diferentes dentro de cada columna son significativamente diferentes según Tukey $(\mathrm{p} \leq 0,05)$.

${ }^{\prime}$ Growth regulator $=1 \mathrm{~mL}$ per plant of a $5 \mathrm{mg} \cdot \mathrm{L}^{-1}$ solution of 6-benzyladenine $(B A)$. Fertilizer $=15 \mathrm{~g}$ per plant of an $N: P: K$ mixture $(15: 15: 15, v: v: v)$.

${ }^{2}$ The interaction between fertilizer and growth regulator was not statistically significant at $p \leq 0.05$, for the number of leaves, fresh leaf weight, and total fresh weight per plant. Means followed by different letters within each column are significantly different according to Tukey $(p \leq 0.05)$.

poseen una alta relación carbono nitrógeno $(\mathrm{C} / \mathrm{N})$. El carbono de reserva se utiliza en la brotación (Divo de Sesar, 2005). Por lo tanto, en la primera medición realizada el 1 de marzo, la brotación fue mayor en las plántulas de mayor diámetro. De este modo, aparentemente se encubrió las respuestas a la citoquinina, a pesar que el número de yemas diferenciadas en esta fecha fue mayor. Esto se evidenció en la medición del 29 de marzo, cuando se observó mayor proporción de hojas de mayor tamaño (Cuadros 2 y 3).

Las plantas tratadas con BAP brotaron anticipadamente durante dos primaveras consecutivas (Figura 1). Si bien las diferencias de brotación entre TF, TFCk y T no fueron estadísticamente significativas, fue notoria la uniformidad de las plantas tratadas. Asimismo, éstas tuvieron un mayor número de hojas de mayor tamaño (Cuadro 3).

Las diferencias en proteína bruta no siempre fueron estadísticamente significativas (Cuadro 4). No obstante, al mantener estas plantas bajo semi sombra, tuvieron menor contenido de materia seca que la media citada en la bibliografía para plantas mantenidas al sol (Fidler, 1999).

El menor porcentaje de proteína en las plantas del tratamiento TCk fue esperable debido a la presencia de hojas de mayor tamaño (Cuadro 2). Por lo tanto, el nitrógeno disponible disminuyó tempranamente, limitando los componentes nitrogenados de la hoja. El porcentaje de fibra insoluble en detergente neutro (FIDN) libre de cenizas, habitualmente se relaciona con la cantidad de materia seca y su digestibilidad. Por lo tanto, fue menor en las plantas del tratamiento TFCk. El análisis de la FIND comprendió todos los componentes de la pared celular (celulosa, hemicelulosa, lignina y sílice). A medida que una planta madura, aumenta su contenido de FIDN, lo que determina una menor tasa de digestión de ésta, con mayor tiempo de tránsito por el tracto digestivo. En términos prácticos, el FIDN es inversamente proporcional a la capacidad de consumo que los animales tienen sobre un alimento. Por lo tanto, a mayor FIDN, menor es el consumo voluntario. En forrajes se estima que valores de FIDN mayores al 60\% disminuyen la digestibilidad del mismo (Fahey 
Cuadro 4. Efecto del regulador (6-benciladenina) de crecimiento y de la fertilización (N:P:K) determinado por análisis foliar, en muestras de hojas de morera (Morus alba) realizados, en cortes de primavera.

Table 4. Effects of the growth regulator (6-benzyladenine) and fertilization (N:P:K) determined by foliar analysis of samples taken from white mulberries (Morus alba) in the spring cut.

\begin{tabular}{lcccc}
\hline & \multicolumn{4}{c}{ Análisis foliar } \\
\cline { 2 - 5 } Tratamientos $^{1}$ & $\begin{array}{c}\text { Materia seca } \\
\%\end{array}$ & $\begin{array}{c}\text { Proteína bruta } \\
\%\end{array}$ & $\begin{array}{c}\text { Cenizas } \\
\%\end{array}$ & $\begin{array}{c}\text { Fibra }^{2} \\
\%\end{array}$ \\
\hline Testigo (T) & $17,81 \mathrm{a}^{3}$ & $17,91 \mathrm{~b}^{3}$ & $10,08 \mathrm{~b}^{3}$ & $47,05 \mathrm{a}^{3}$ \\
Regulador de crecimiento (TCk) & $18,00 \mathrm{a}$ & $21,74 \mathrm{~b}$ & $13,28 \mathrm{a}$ & $46,67 \mathrm{a}$ \\
Fertilizante (TF) & $18,85 \mathrm{a}$ & $23,39 \mathrm{a}$ & $11,40 \mathrm{ab}$ & $38,54 \mathrm{~b}$ \\
TCk + TF & $17,9 \mathrm{a}$ & $26,13 \mathrm{a}$ & $12,74 \mathrm{a}$ & $29,73 \mathrm{c}$ \\
\hline
\end{tabular}

${ }^{1}$ Regulador de crecimiento $=1 \mathrm{~mL}$ por planta de una solución de 6-bencilaminopurina $(\mathrm{BAP})\left(5 \mathrm{mg} \cdot \mathrm{L}^{-1}\right)$. Fertilizante $=15 \mathrm{~g}$ de $\mathrm{N}: \mathrm{P}: \mathrm{K}$ (15:15:15, v:v:v:).

${ }^{2}$ Fibra determinada en detergente neutro libre de cenizas.

${ }^{3}$ Cada valor es el promedio de cinco repeticiones. La interacción entre fertilizante y regulador de crecimiento no fue estadísticamente significativa a $\mathrm{p} \leq 0,05$. Promedios seguidos por letras diferentes dentro de cada columna son significativamente diferentes según Tukey $(\mathrm{p} \leq 0,05)$.

${ }^{1}$ Growthregulator $=1 \mathrm{mLperplantofa} 5 \mathrm{mg} \cdot \mathrm{L}^{-1}$ solutionof6-benzyladenine $(B A)$. Fertilizer $=15 \mathrm{~g}$ perplant of an $N: P:$ Kmixture $(15: 15: 15, v: v: v)$. ${ }^{2}$ Fiber was determined in ash-free, neutral detergent.

${ }^{3}$ Each value is the mean of five replicates. The interaction between fertilizer

and growth regulator was not statistically significant at $p \leq 0.05$. Means followed by different letters within each column are significantly different according to Tukey $(p \leq 0.05)$.

y Berger, 1988). El gusano de seda sólo utiliza para su alimentación el parénquima foliar.

\section{Bioensayo con gusano de seda}

Los resultados obtenidos en los ensayos realizados se observan en la Figura 2. Hubo diferencias significativas $(\mathrm{p} \leq 0,05)$ en la respuesta a la alimentación diferencial en las dos fechas evaluadas en el año. Los resultados del bioensayo realizado en otoño demostraron la existencia de un efecto positivo de TF sobre el peso de las larvas, obteniéndose ganancias promedio de $13,94 \pm 0,03 \mathrm{~g}$.

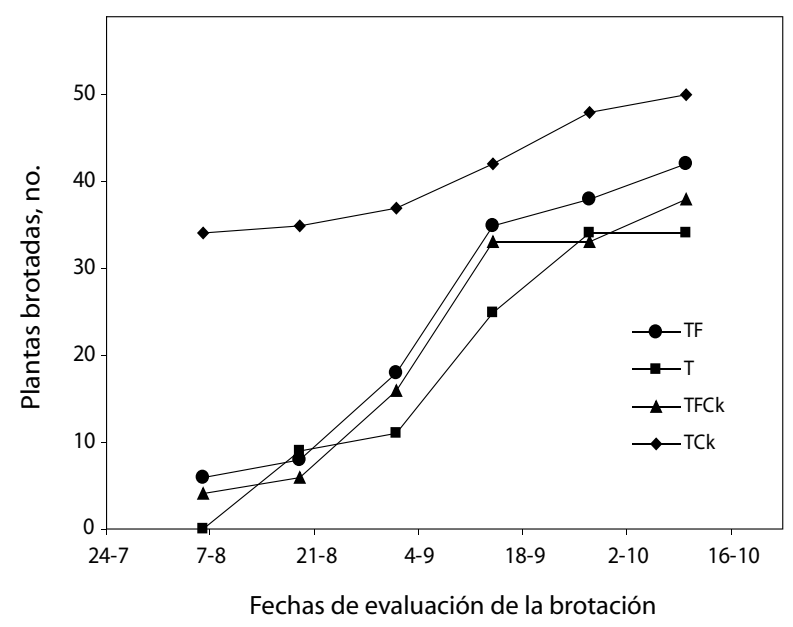

Figura 1.Efecto del regulador (6-benciladenina) de crecimiento y de la fertilización (N:P:K) sobre la eevolución del número de moreras (Morus alba) brotadas en función del tiempo. TF= plantas fertilizadas con N:P:K, TCk=plantas tratadas con regulador decrecimiento, $\mathrm{TFCk}=$ plantasfertilizadasytratadasconreguladordecrecimientoyT=plantastestigos. Seindicadía-mes. $(\mathrm{n}=55)$. Figure 1. Effect of the growth regulator (6-benzyladenine) and fertilization (N:P:K) on the number of white mulberry (Morus alba) plants with open buds as function of time. $T F=$ plants fertilized with $N: P: K, T C k=$ plants treated with growth regulator, $T F C k=$ plantsfertilizedandtreated with growthregulator, and $T=$ untreatedplants.Datesareindicatedasday-months. $(n=55)$. 


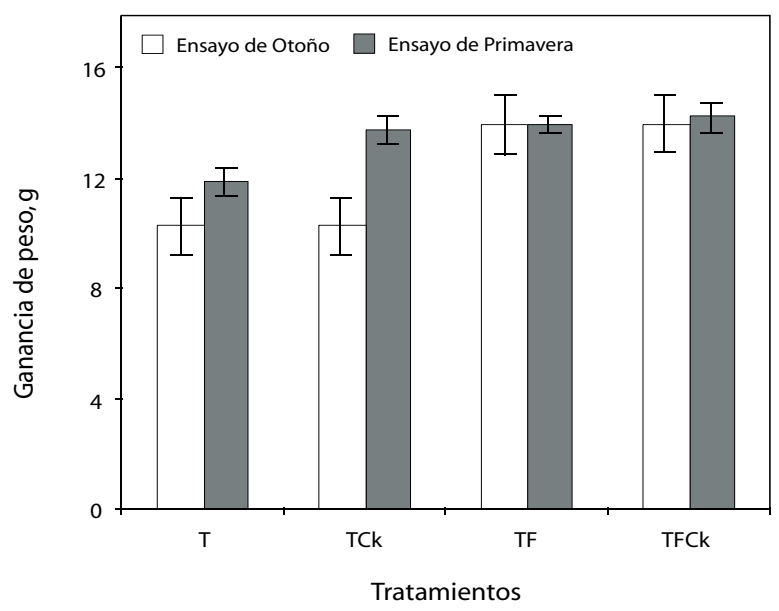

Figura 2: Ganancia de peso de larvas de gusano de seda (Bombyx mori) alimentadas con hojas de morera (Morus alba) provenientes de plantas tratadas con diferentes combinaciones de reguladores de crecimiento y/o fertilizante, determinado en bioensayos realizados en otoño y primavera. $\mathrm{TF}=$ plantas fertilizadas con $\mathrm{N}: \mathrm{P}: \mathrm{K}, \mathrm{TCk}=$ plantas tratadas con regulador de crecimiento, $\mathrm{TFCk}=$ plantas fertilizadas y tratadas con regulador de crecimiento (6-benciladenina) y $\mathrm{T}=$ plantas testigos. Barras = desviación estándar.

Figure 2. Weight gain by larvae of silkworms (Bombyx mori) fed with leaves of white mulberry (Morus alba) plants treated with different combinations of growth regulator and/or fertilizer in a bioassay performed in autumn (white bars) and spring (gray bars). $T F=$ plants fertilized with $N: P: K, T C k=$ plants treated with growth regulator (6-benzyladenine), TFCk $=$ plants fertilized and treated with growth regulator, and $T=$ untreated plants. Bars $=$ standard deviation .

En los primeros días, se observó que el tratamiento con BAP afectó negativamente a las larvas. Estas se retorcían, evacuando el intestino y consumiendo menor cantidad de alimento. Si bien este efecto duró 2 días y no se presentó en el ensayo primaveral, afectó el peso total del otoño $(3,92 \pm 0,01 \mathrm{~g})$. Aún cuando el consumo y comportamiento posterior se normalizó, el estrés resultante impidió que las larvas de este tratamiento alcanzaran el desempeño obtenido con larvas sometidas a los otros tratamientos. Existen antecedentes respecto de la actividad antialimentaria de las citoquininas sobre lepidópteros. Esto ocurre en plantas que sobreexpresan el gen de las mismas y/o se suplementan con BAP. Como en larvas de Manduca sexta L. (Lepidoptera: Esfingidae), que alimentadas con hojas de tabaco transformadas disminuyeron su ingesta en un 70\% (Smigocki et al., 1993; Smigocki et al., 2000). Por lo tanto, ello debe considerarse al momento de implementar un programa de cría a nivel industrial. El efecto residual de los tratamientos con citoquininas dura aproximadamente 3 a 4 meses según se ha determinado para otros especies vegetales (Divo de Sesar, 2005).
En el bioensayo primaveral se obtuvieron diferencias estadísticamente significativas entre T y TF y TFCk, sin observar el efecto negativo evidenciado en el ensayo otoñal en las larvas alimentadas con hojas provenientes del tratamiento TCk, indicado anteriormente.

En la Figura 3 se muestra el porcentaje de seda bruta obtenida para los distintos tratamientos. $\mathrm{Si}$ bien existen diferencias significativas en el porcentaje de seda bruta entre tratamientos y el testigo (T), no se observó un aumento de similar magnitud al de la ganancia en peso de las larvas (Figura 2). Probablemente ello se debió a que el porcentaje de seda bruta obedece a múltiples factores. Además de la calidad de la alimentación depende también de la raza del gusano de seda, sexo, maduración de la pupa, tiempo de cría, contenido de agua en la corteza del capullo (Cifuentes y Shon, 1998).

El apetito del gusano de seda varía según la raza, edad de desarrollo, condiciones ambientales y calidad de la morera. Aunque el mayor consumo foliar lo efectúa en el cuarto y quinto estadio larval, la asimilación es más alta en el 


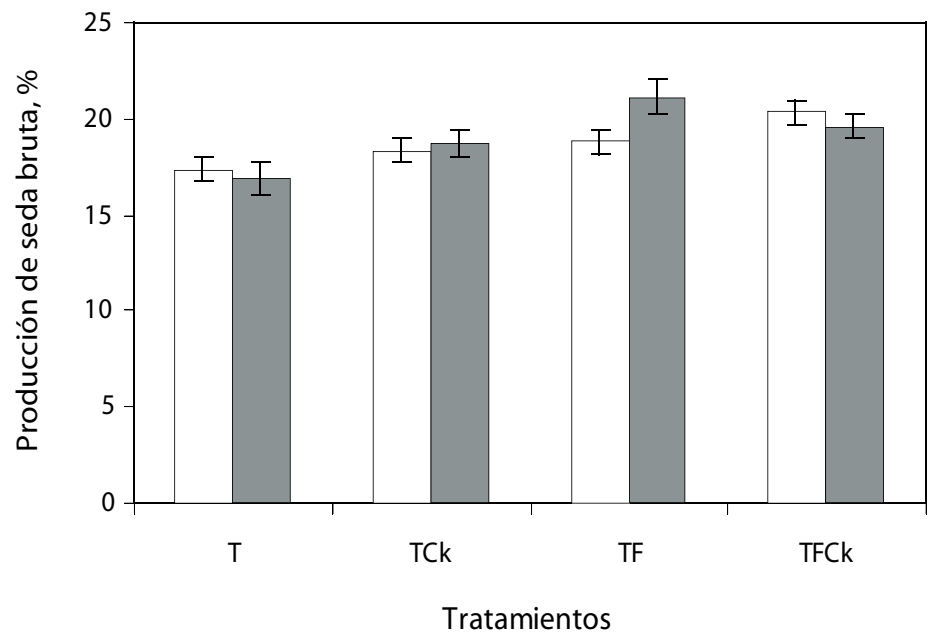

Figura 3. Producción de seda bruta en en capullos de gusano de seda (Bombyx mori) alimentados con hojas de morera (Morus alba) sometidas a diferentes tratamientos. Los bioensayos se realizaron en otoño (barras blancas) y en primavera (barras grises). $\mathrm{TF}=$ plantas fertilizadas con $\mathrm{N}: \mathrm{P}: \mathrm{K}, \mathrm{TCk}=$ plantas tratadas con regulador de crecimiento, $\mathrm{TFCk}=$ plantas fertilizadas y tratadas con regulador de crecimiento (6-benciladenina) y T = plantas testigos. Barras = desviación estándar. Figure 3. Raw silk produced by the silkworm (Bombyx mori) cocoons fed on leaves of white mulberry (Morus alba) plants subjected to different treatments. Assays were performed in autumn (white bars) and spring (gray bars). TF = plants fertilized with N:P:K, TCk = plants treated with growth regulator, TFCk = plants fertilized and treated with growth regulator, and $T=$ untreated plants. Bars $=$ standard deviation.

primero (65.7\%) que en el último (48.5\%), por lo que la calidad del alimento es fundamental en la primeras etapas de desarrollo, ya que la utilización de hojas de moreras de baja calidad puede provocar una mortalidad de alrededor del $80 \%$, mientras que empleando alimentos de calidad (mayor porcentaje de proteína) ésta disminuye al 16\% (Cifuentes y Sohn, 1998).

Los porcentajes de seda cruda obtenidos en estos ensayos (Figura 3) son coincidentes con los estándares actuales de las razas de gusanos de seda que se comercializan mundialmente (17\% a 24\%) (Cifuentes y Shon, 1998). Si bien, el sistema de clasificación y análisis de capullos frescos utilizado para la fijación del precio a los productores, se basa en tres puntos básicos: contenido de seda bruta, proporción devanable de los capullos, tamaño y peso de cada capullo (Cifuentes y Kim, 1992). No obstante, el primer factor tiene mayor incidencia en la determinación del valor comercial (50\%). Por lo tanto, un aumento en los valores de seda cruda, redunda significativamente en la rentabilidad.
Se concluye que la utilización de combinación de reguladores de crecimiento y fertilizantes permite obtenerplantas con ciclos decrecimiento más prolongados, mayor productividad y calidad de la materia verde. Esto afecta positivamente la ganancia de peso de las larvas y el porcentaje de seda bruta obtenido.

\section{Resumen}

El objetivo de este trabajo fue determinar un protocolo eficiente para la propagación vegetativa de la morera (Morus alba). Al mismo tiempo tuvo el propósito de evaluar la influencia de este protocolo de propagación en la alimentación de las larvas de Bombyx mori y en el porcentaje de seda cruda producido. Plantas de morera, propagadas vegetativamente, fueron transplantadas a macetas de $4 \mathrm{~L}$, tratadas con N:P:K (15:15:15, $15 \mathrm{~g})(\mathrm{F})$ y/o 6-bencilaminopurina $\left(5 \mathrm{mg} \cdot \mathrm{L}^{-1}\right)$ (Ck). En otoño y primavera, se utilizaron hojas para la alimentación de una cohorte de larvas de tercer estadio. Se observó que el $60 \%$ de las plantas tratadas con $\mathrm{Ck}$ brotaron en julio 
y las restantes lo hicieron en agosto. Aunque las plantas fertilizadas (TF) y fertilizadas y tratadas con 6-bencilaminopurina (TFCk), brotaron junto al testigo $(\mathrm{T})$, presentaron mayor número de hojas de mayor tamaño. En TFCk se observó menor porcentaje de FIDN (29,73). La calidad del alimento se reflejó en la ganancia de peso y el porcentaje de seda cruda producido. Si bien no se observó interacción entre $\mathrm{F}$ y $\mathrm{Ck}$, se encontraron diferencias significativas entre las plantas testigos (T) y TF, TCk y TFCk. En el ensayo de otoño, el tratamiento TCk afectó negativamente a las larvas de $B$. mori provocando retorcimiento e induciendo una asidua evacuación intestinal. Esto provocó una menor ingesta de alimento, efecto transitorio, pero que resultó en un menor peso final. Los resultados de este trabajo son muy promisorios pudiendo ser útiles para producciones masivas, ya que una prolongación en el ciclo vegetativo permitiría mayor número de generaciones por año.

Palabras clave: Bombyx mori, citoquinina, Morus alba.

\section{Agradecimientos}

Este trabajo se realizó con fondos provenientes del proyecto UBACyT G 106 de la Universidad de Buenos Aires.

\section{Literatura citada}

Acerbi, M., G. Mozeris, C. y Vieites, C. M. 2005. Análisis FODA del sistema sericícola argentino. Revista Facultad de Agronomía. Universidad de Buenos Aires 25:31-39.

Cifuentes, C. A. y Kim, M. H. 1994. Sistema "Cokosilk" de clasificación de capullos. En: Sericultura Colombiana. Publicación del Centro de Desarrollo Tecnológico de Sericicultura (CDTS)1,3:11-13.

Cifuentes, C. A. y K.W. Schon. 1998. Manual Técnico de Sericicultura. Primera ed. Centro de Desarrollo Tecnológico de Sericicultura (CDTS). Ciudad de Risaralda, Colombia. 438 pp.

Di Rienzo,J.A., A.W. Guzmán, and F. Casanoves. 2002. A multiple comparisons method based on the distribution of the root node distance of a binary tree. Journal of Agricultural, Biological, and Environment Statistics 7:1-14.

Divo de Sesar, M. 2005. Integración de estudios fisiológicos, histológicos y bioquímicos del proceso de enraizamiento, rustificación y crecimiento posterior de especies de importancias agronómica suplementadas con citoquininas. Tesis Doctoral. Universidad de Buenos Aires. Buenos Aires, Argentina. 221 pp.

Divo de Sesar, M., A. D'Ambrogio, J. Boquete, F. Vilella y A. Stella. 2003. Determinación del momento óptimo de aplicación de 6-bencilaminopurina y correspondencia con la secuencia histológica en el proceso de enraizamiento de estacas semileñosas de Jasminum mensyi Hance (Oleaceae). Páginas 27-36, En: A.J. Pascale (ed.), Floricultura en la Argentina, Investigación y Tecnología de Producción. Editorial Facultad de Agronomía, Universidad de Buenos Aires, Argentina. 468 pp.

Fahey, G., and L. Berger. 1988. Carbohydrate nutrition in ruminants. Pages 269-297, In: D.C. Church (ed.), The Ruminant Animal, Digestive Physiology and Nutritition. Prentice Hall, New Yersey, USA.

Fidler, A. 1999. Pot-in-por system: A container grown in the ground approach for diverse crops. Com. Proc. Int. Plant Prop. Soc. 49:297-99.

Hintze, J. L. 1991. Number Cruncher Statistical System (NCSS). Student section S2. Published by Dr Jerry Hintze. Kaysville, Utah, EUA, 167 pp.

Pelicano, A., G. Mareggiani, E. Plante y N. Zamuner. 2004. Calidad de hojas de morera y su influencia en la cría del gusano de seda Bombyx mori (Lep. Bomb). IDESIA (Chile) 22:49-53.

Smigocki, A., S. Heu, and G. Buta. 2000. Analysis of insecticidal activity in transgenic plants carrying the ipt plant growth hormone gene. Acta-Physiologiae-Plantarum. 22:295-299.

Smigocki A., J.W. Jr. Nea, I. McCanna, and L. Douglass. 1993. Cytokinin-mediated insect resistance in Nicotiana plants transformed with the ipt gene. Biology 23:325-335.

Thomas, H., H.J. Ougham, C. Wagstaff, and A. Stead. 2003 Defining senescence and death. Journal of Experimental Botany 54:1127-1132.

Van Soest, P.J., J.B. Robertson, and B. A. Lewis. 1991. Methods for dietary fiber, neutral detergent fiber and non-starch polysaccharides in relation to animal nutrition. J. Dairy Sci. 74:3583-3597. 